\title{
Cordycepin induces apoptosis in SGC-7901 cells through mitochondrial extrinsic phosphorylation of PI3K/Akt by generating ROS
}

\author{
MOUSSA IDE NASSER ${ }^{1}$, MUQADDAS MASOOD $^{1}$, WEI WEI $^{2}$, XIAOCHUN LI $^{1}$, \\ YIFA ZHOU $^{1}$, BAO LIU ${ }^{1}$, JIANG $\mathrm{LI}^{2}$ and XIAOMENG LI ${ }^{1}$
}

\begin{abstract}
${ }^{1}$ The Key Laboratory of Molecular Epigenetics of MOE, Institute of Genetics and Cytology, Northeast Normal University, Changchun, Jilin 130024; ${ }^{2}$ Dental Hospital, Jilin University, Changchun, Jilin 130021, P.R. China
\end{abstract}

Received August 9, 2016; Accepted November 23, 2016

DOI: $10.3892 /$ ijo.2017.3862

\begin{abstract}
Medicinal plants are affluent sources of several effectual natural drugs. Among them cordycepin which is extracted from Cordyceps militaris is a hopeful chemotherapy agent due to its extensive anti-inflammatory, anti-proliferative, antioxidant, and antitumor characteristics. This study investigated the efficacy of cordycepin in the context of human gastric cancer SGC-7901 and searched for the cell death procedure. Cordycepin incorporates mitochondrial-mediated apoptosis in SGC-7901 cells with the help of regulating mitochondrial extrinsic pathways by inhibition of $\mathrm{A}_{3} \mathrm{AR}$ and drive activation of DR3, which promote the activation of PI3K/Akt protein expression as well as collapse of mitochondrial membrane potential (MMP). In addition, phosphorylation of PI3K/Akt and DNA damage by cordycepin induced the production of reactive oxygen species (ROS), and mediates SGC-7901 cell cycle cessation at $\mathrm{S}$ phase. Collectively, this study suggests that cordycepin might be effective as a modern chemotherapy drug for gastric cancer.
\end{abstract}

\section{Introduction}

Gastric cancer (GC) is considered to be one of the most common grave malignancies with high mortality rate worldwide, and particularly in China (1). This is due to its high drug resistance along with the unavailability of proficient diagnostic tools (2). In the past few decades, great efforts

Correspondence to: Professor Xiaomeng Li, The Key Laboratory of Molecular Epigenetics of MOE, Institute of Genetics and Cytology, Northeast Normal University, Changchun, Jilin 130024, P.R. China

E-mail: lixm441@nenu.edu.cn

Professor Jiang Li, Dental Hospital, Jilin University, Changchun, Jilin 130021, P.R. China

E-mail: ljiang@jlu.edu.cn

Key words: cordycepin, SCG-7901, PI3K/Akt, apoptosis were made to explore cogent therapeutic approaches for GC including chemotherapy (3) as well as immunotherapy (4). Natural drugs for example, hispolon, lentinan, and grifolin derived from different mushrooms showcased a highly therapeutic effect to curb GC. Of great interest is that these natural compounds do not possess any side effects on bone marrow, blood haemoglobin, and immune system in respect of traditional therapeutic methods (5-7).

Cordycepin an adenosine analogue, extracted from Cordyceps militaris mushroom has gained attention with its anti-inflammatory, antioxidant, and antibacterial features $(8,9)$. Currently, tumor genesis of cordycepin has been presented in many types of cancers. For example, cordycepin integrates inhibition of the proliferation of lung and renal cancer cells through mediating their cell death due to apoptosis $(10,11)$. Apoptosis (programmed cell death) is a stern mechanism of physiological cell death, that keeps up cell proliferation and homoeostasis in tissue (12). Furthermore, the processes through which cordycepin mediated apoptosis was presented to be by mitochondrial extrinsic pathways (13). The mitochondrial extrinsic apoptosis is monitored by death receptors like DR3 that, once activated, trigger activation of caspase- 8 that proceeds with mediating the activation of caspase-3 as well as cleaved PARP (14). Death receptors have a direct apoptotic pathway, transfer apoptosis signals (from death receptor) to death ligands, and consequently play an important role in instructive apoptosis (15). These receptors can activate death caspases within seconds of ligand binding, causing an apoptotic demise of the cell within hours (15). Generally, DR3 regulate inflammation and autoimmune diseases: experimental autoimmune uveoretinitis, allergic lung inflammation and inflammatory arthritis $(16,17)$. Cordycepin was used to treated gastric cancer EBV-positive (SNU-791 cell line), without affecting their cell proliferation or cell cycle arrest (18). Interestingly, the combination of cordycepin with doxorubicin enhanced gastric cancer (AGS cell line) EBV-positive cell proliferation notably by p38 MAPK signalig pathways (19). Moreover, cordycepin also mediated apoptosis with the help of mitochondrial intrinsic pathways by inhibiting PI3K/Akt signaling pathway and generating ROS $(20,21)$. 
PI3K/Akt plays a potential role in several cancer cells by regulating cell proliferation, survival and metabolism. It is reported that phosphorylarylation of PI3K/Akt leads to inhibition of gastric cancer cell proliferation (22). In the same manner, activation of Akt results into inhibition of MDM2 and generation of $\mathrm{p} 53$, incorporating gastric cancer cell proliferation arrest (23). p53 also induces upregulation of the Bax and downregulation of Bcl-2 during p53-related apoptosis (24). In addition, the inhibition of Akt is also linked to the production of ROS in SGC-7901 cells (25). ROS is a product of oxygen metabolism following cellular stress. In accordance with its generation, ROS can either possess positive or negative influence on cells. Many cancer cells generate a moderate level of ROS to sustain their proliferation, migration and proliferation. Furthermore, most of the gastric cancer cells pose resistance to chemotherapy drugs quite clearly due to their moderate production of ROS. As a result, extreme levels of ROS bring forth adverse environment thereby promoting cells death by apoptosis $(26,27)$.

Although, previous studies underline the mechanism by which cordycepin regulates many cancers, its mechanism in gastric cancer is still unclear. We performeded an in vitro study to observe the anti-proliferative result of cordycepin, Cordyceps militaris, mushroom, on the gastric cancer SGC-7901 cells. SGC-7901 cells (EBU_) are frequently investigated representative gastric cancer cell line. Aim of this study was to demonstrate the effect of cordycepin on cancer cells and the molecular pathway by which it induces apoptosis and prevent cell survival signal in human gastric cancer SGC-7901 cells. We also verified the role of cordycepin as ROS inducer promoting selective killing of cancer cells.

\section{Materials and methods}

Chemicals and reagents. Cordycepin was purchased from Chengdu Pufei De Biotech Co., Ltd. (China). Fetal bovine serums was purchased from Hangzhou Sijiqing Biological Engineering Materials Co. (China). Dulbecco's modified Eagle's medium (DMEM) were purchased from (Gibco, China), 3-4,5-dimethylthiazol-2-yl-2,5-diphenyltetrazolium bromide (MTT), and dimethyl sulfoxide (DMSO) were from Sigma Chemical Co. (St. Louis, MO, USA). Propidium iodide (PI), Annexin V-FITC Apoptosis Detection kit, rhodamine 123 and reactive oxygen species kit were from Beyotime Institute of Biotechnology (Shanghai, China). Rabbit polyclonal antihuman Bcl-2, anti-human Bax, P-PI3K, Akt, P-Akt, caspase-8, antibodies were purchased from Cell Signaling Technology (Beverly, MA, USA). Mouse, anti-rabbit, caspase-3 p-JAK2 (catalog no. bs-2485R), p-Stat3 (catalog no. bs-1658R) were purchased from Bioss (Beijing Biosynthesis Biotechnology), p53, MDM2, survivin, Cdk2, Cdk1, and cyclin E antibodies were purchased from Santa Cruz Biotechnology, DR3, A AR antibodies were purchased from Kanghexin Biotech Co. (Suzhou, China).

Ethics/guidelines. We followed the ethical classification standards and our laboratory guidelines; SGC-7901 (gastric cancer) cell line was purchased from ATCC (Shanghai, China).
Cell culture. SGC-7901 cells were seeded and cultured in 10-cm dish with DMEM medium contained 10\% FBS (Gibco), then incubated in an incubator (humidified $37^{\circ} \mathrm{C}$, the atmosphere of $5 \% \mathrm{CO}_{2}$ ). Cells were allowed to grow to $70-80 \%$ before used.

Determination of cell cytotoxicity by MTT. SGC-7901 cells were seed and cultured in a 96-well plate to a final concentration of $5 \times 10^{3}$ with DMEM medium with $1 \%$ FBS then incubated at $37^{\circ} \mathrm{C}$ for $18-24 \mathrm{~h}$. After all, cells were treated with the appropriated concentration of cordycepin for $24 \mathrm{~h}$. Afterward, the cell medium was discarded, and fresh medium was added to each well, with $20 \mu \mathrm{l}$ MTT solutions $(5 \mathrm{mg} / \mathrm{ml})$, and then incubated at $37^{\circ} \mathrm{C}$ for $4 \mathrm{~h}$. Finally, $150 \mu \mathrm{l}$ of DMSO was added to each well, incubated in the dark for $10 \mathrm{~min}$, then read at the wavelength of $570 \mathrm{~nm}$ using Varioskan Flash Multimode Reader (Thermo Scientific, USA). The absorbance was measured at $570 \mathrm{~nm}$, and results were expressed as a percentage, relative to solvent-control incubations, and the $\mathrm{IC}_{50}$ were determined using non-linear regression analysis (percentage survival versus concentration).

Annexin V/PI assays for apoptosis. For Annexin V/PI assays, SCG-7901 cells were stained with Annexin V-FITC and PI, and evaluated for apoptosis by flow cytometry according to the manufacturer's protocol (Beyotime, China). After treatment with $0,20,40$ and $80 \mu \mathrm{M}$ of cordycepin, and incubation at $37^{\circ} \mathrm{C}$ for $24 \mathrm{~h}, \mathrm{SGC}-7901$ cells were collect and washed twice with PBS, then stained with $5 \mu \mathrm{l}$ of Annexin V-FITC and $10 \mu \mathrm{l}$ of PI in $500 \mu \mathrm{l}$ binding buffer for $15 \mathrm{~min}$ at room temperature in the dark. The apoptotic cells were determined using flow cytometry, and the data were analysed using CellQuest analysis software.

DAPI (4',6-diamidino-2-phenylindole) staining. SGC-7901 cells were cultured in 12-well plates. After $24 \mathrm{~h}$, cells were treated with cordycepin for $24 \mathrm{~h}$, and then fixed in $4 \%$ cold paraformaldehyde (PFA) for $30 \mathrm{~min}$. Subsequently, SGC-7901 cells were incubated with high DAPI $(1 \mathrm{mg} / \mathrm{ml})$ for $30 \mathrm{~min}$ in the dark; then, the cells were washed with PBS. Apoptotic nuclei characterized as intensively stained were detected using fluorescent microscopy (model IX71; Olympus, Tokyo, Japan).

Cell cycle analysis. The cell cycle distribution in different phases after exposure of cordycepin were analysed by flow cytometry. In brief, SGC-7901 cells were harvested and washed with PBS after exposure to $0,20,40$ and $80 \mu \mathrm{M}$ of cordycepin for $24 \mathrm{~h}$. Subsequently, the cells were fixed with $70 \%$ of ethanol at $-20^{\circ} \mathrm{C}$ for $2 \mathrm{~h}$ and then stained with PI solution consisting of $1 \mathrm{mg} / \mathrm{ml} \mathrm{PI}$ and RNase A. The fluorescence-activated cells were analysed by the flow cytometry, and the data were analyzed using CellQuest analysis software.

Determination of intracellular reactive oxygen species (ROS) production. The ROS generation was measured after staining the cells with DCFH-DA and flow cytometry. Briefly, after exposure of different concentrations of cordycepin, SGC-7901 stained by $1 \mathrm{ml}$ of PBS containing $10 \mu \mathrm{M}$ DCFH-DA, and 
incubated for $30 \mathrm{~min}$ at $37^{\circ} \mathrm{C}$. The fluorescence emission from DCF was analysed via CellQuest analysis software flow cytometry (Cytomics FC 500; Beckman Coulter Inc., Miami, FL, USA), with excitation and emission spectra set at 480 and $530 \mathrm{~nm}$, respectively.

Determination of mitochondrial trans-membrane potential $(M M P)$. The MMP was measured using a flow cytometry (Cytomics FC 500; Beckman Coulter Inc.), and the fluorescent dye rhodamine 123. In brief SGC-7901 cells were cultured in 12-well plates, after treatment with cordycepin cells were harvested, and washed with PBS. Then stained with Rh123 $(100 \mu \mathrm{g} / \mathrm{l})$ for $30 \mathrm{~min}$ at $37^{\circ} \mathrm{C}$. The cells were collected by pipetting and washed twice with PBS and analysed by flow cytometry.

Western blot analysis. SGC-7901 cells were treated with 0, 20, 40 and $80 \mu \mathrm{M}$ cordycepin in DMEM medium with $1 \%$ FBS incubated for $24 \mathrm{~h}$. The cells were harvested and collected on ice-cold PBS; further RIPA containing proteinase inhibitor cocktail was added in cells, incubated on ice for $30 \mathrm{~min}$. Afterward, insoluble protein lysate was removed by centrifugation at $1,350 \mathrm{rpm}$ for $15 \mathrm{~min}$ at $4^{\circ} \mathrm{C}$. The protein concentrations were measured by using NanoDrop 1000 (Thermo Scientific) spectrophotometer, $70 \mu \mathrm{g}$ of proteins were resolved on $10-12 \%$ SDS-PAGE and transferred to PVDF membranes. After blocking with $5 \%(\mathrm{w} / \mathrm{v})$ non-fat milk and washing with a Tris-buffered saline-Tween solution (TBST), membranes were incubated with respective primary antibodies at $4^{\circ} \mathrm{C}$ overnight and washed three times with TBST. The blots were then incubated with anti-rabbit or anti-mouse horseradish peroxidase conjugated secondary antibodies for $1 \mathrm{~h}$ at room temperature. Finally membrane was washed again with TBST three times; signals were detected using ECL plus chemiluminescence kit on X-ray film (Millipore Corp., Billerica, USA) (28).

Statistical analysis. Statistical analysis was performed using Origin lab 8. Each experiment was repeated at least three times. All data are presented as the mean \pm standard deviation (SD). Statistical significance was evaluated using one-way analysis of variance (ANOVA). Differences were considered to be statistically significant at $\mathrm{P}<0.05$.

\section{Results}

Cytotoxicity of cordycepin. MTT assay was used to assess the cell viability of SGC-7901 cells in the presence of several concentrations of cordycepin (Fig. 1A) ranging from 0 to $100 \mu \mathrm{M}$. The growth inhibition improved consistently with time over the period of the incubation time. Specifically, the estimated half-maximal inhibitory concentration $\left(\mathrm{IC}_{50}\right)$ values were 40,32 and $7 \mu \mathrm{M}$ after 24,48 and $72 \mathrm{~h}$, correspondingly (Fig. 1B). The cytotoxicity of cordycepin was further observed after the SGC-7901 cells were treated with 0, 20,40 and $80 \mu \mathrm{M}$ cordycepin. As seen in Fig. 1C cordycepin inhibited SGC-7901 cell proliferation and cells death rate increased with incensement of cordycepin concentration.

Cordycepin induces apoptosis in SGC-7901 cells. Loss of membrane plasma and DNA fragmentation are the key characteristics possessed by apoptotic cell death. The impact of cordycepin on SGC-7901 cells death was evaluated by DNA fragmentation with the help of DAPI staining and fluorescent microscopy. As seen in Fig. 2A cordycepin incorporates the shape of the SGC-7901 cells modified to a considerable extent via increasing dose-dependently. Particularly, cordycepin broke the cell membranes leading to inducing the nuclear condensation by apoptotic in comparison with the control cells. Induction of apoptosis was further validated by Annexin V/PI assay. This is based on the probe of the initial apoptosis (B4), late apoptosis (B2), and necrosis (B1) of SGC-7901 cells. The results indicated that the B4 values increased $8.091 \pm 1.435$, $24.37 \pm 1.829,49.33 \pm 1,492$ and $89.74 \pm 2.714 \%$ by utilizing 0,20 , 40 and $80 \mu \mathrm{M}$ of cordycepin, correspondingly (Fig. 2B).

Additionally, to evaluate whether cordycepin-induced apoptosis was dependent upon mitochondrial pathways, western blot analysis was carried out to monitor protein expression of mitochondrial extrinsic apoptosis. As shown in Fig. 2C, treatment of SGC-7901 cells by cordycepin, induces activation of death receptor DR3. Indeed, in the absence of cordycepin DR3 protein expression is null while increased together with the cordycepin concentration. This activation of DR3 encouraging activation of caspase-8, that functions as an initiator of caspase-3, further enhancing cleavage of PARP consequently inducing SGC-7901 cell death by apoptosis (14).

Cordycepin induces SGC-7901 cell apoptosis by inhibiting PI3K/AKT Pkm2, and increases, p38 in SGC-7901 cells. The phosphatidylinositol 3-kinase/Akt pathways are engaged in the SGC-7901 tumor expansion and its metastasis. Especially, the inhibition of Akt results in disturbance of the biological activities of SGC-7901 cells by mediating their cell cycle arrest (29). A previous report, supported that the activation of DR3 in colon cancer cells, leads to activation of PI3K inducing cell apoptosis (30). We therefore, evaluated whether the activation of DR3 in SGC-7901 cells was associated with activation of PI3K/Akt signaling. As presented in Fig. 3, cordycepin inhibits the expression level of PI3K, considerably decreasing P-Akt protein expression. At the same time, cordycepin boosts the expression of p38 whereas Akt remains almost the same. These findings show that cordycepin is likely to induce SGC-7901 death by mediating their cells cycle arrest, and this through inhibition of AKT and increased p38.

PKM2 is a key enzyme that regulates aerobic glycolysis in tumor cells and particularly in gastric cancer. Its inhibition was report to affect SGC-7901 cells growth (31). We therefore, investigated the expression of PKM2 in SGC-7901 cells. As shown in Fig. 5, cordycepin suppressed PKM2 expression. We can therefore suggest that this inhibition of PKM2 by cordycepin contributed to the induction apoptosis in SGC-7901 cells.

Cordycepin induces SGC-7901 cell apoptosis by inhibition of $A_{3} A R . \mathrm{A}_{3} \mathrm{AR}$ is a normal purine metabolite extensively expressed in most cancer cells. Its inhibition is involved in inhibiting the proliferation and induction of cell death by apoptosis (32). To gauge whether the apoptosis mediated by cordycepin possesses the capacity to influence $\mathrm{A}_{3} \mathrm{AR}$ expression in SGC-7901 cells western blot analysis was carried 
<smiles>Nc1ncnc2c1ncn2[C@@H]1O[C@@H](CO)C[C@H]1O</smiles>

C

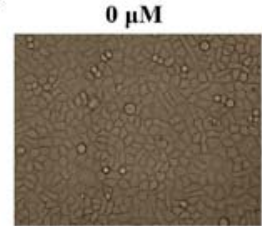

$40 \mu \mathrm{M}$

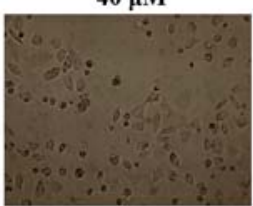

B

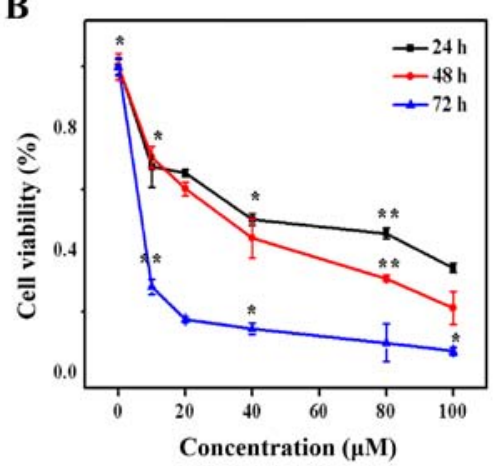

$20 \mu \mathrm{M}$

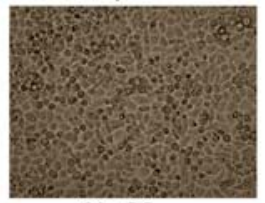

$80 \mu \mathrm{M}$

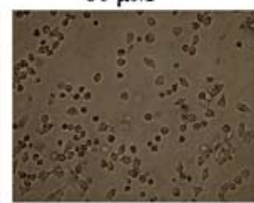

Figure 1. Effect of cordycepin on SGC-7901 cell morphology and viability. (A) Structure of cordycepin. (B) SGC-7901 cells were treated with 0, 10, 20, 40, 80 and $100 \mu \mathrm{M}$, Proliferation was assessed after 24, 48, and $72 \mathrm{~h}$, as described in Materials and methods. Each bar represents the mean \pm standard deviation of three experiments. (C) Morphological changes of SGC-7901 cells were observed by phase-contrast microscopy, control (without cordycepin treatment) and with 20,40 and $80 \mu \mathrm{M}$ of cordycepin for $24 \mathrm{~h}$.
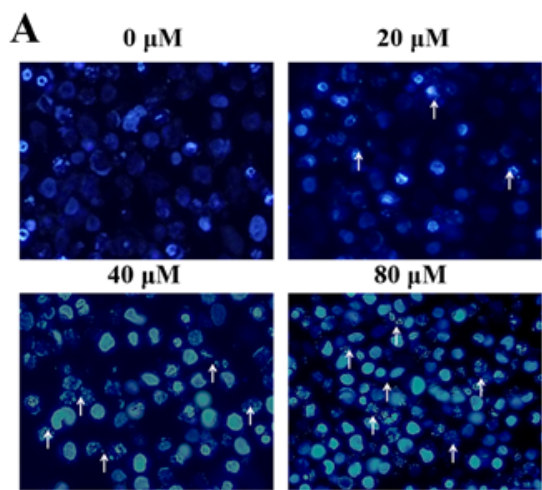

C

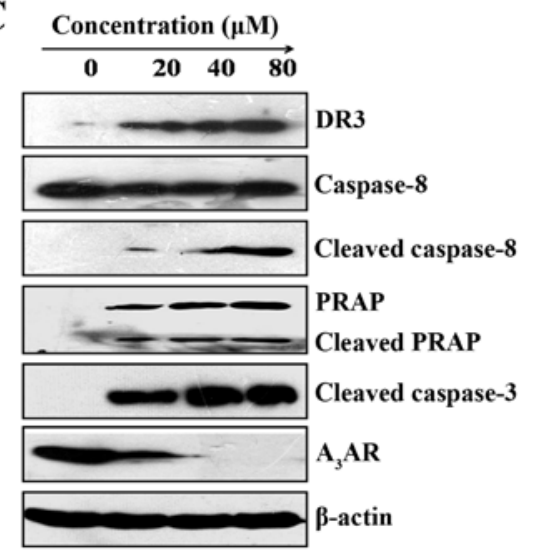

B
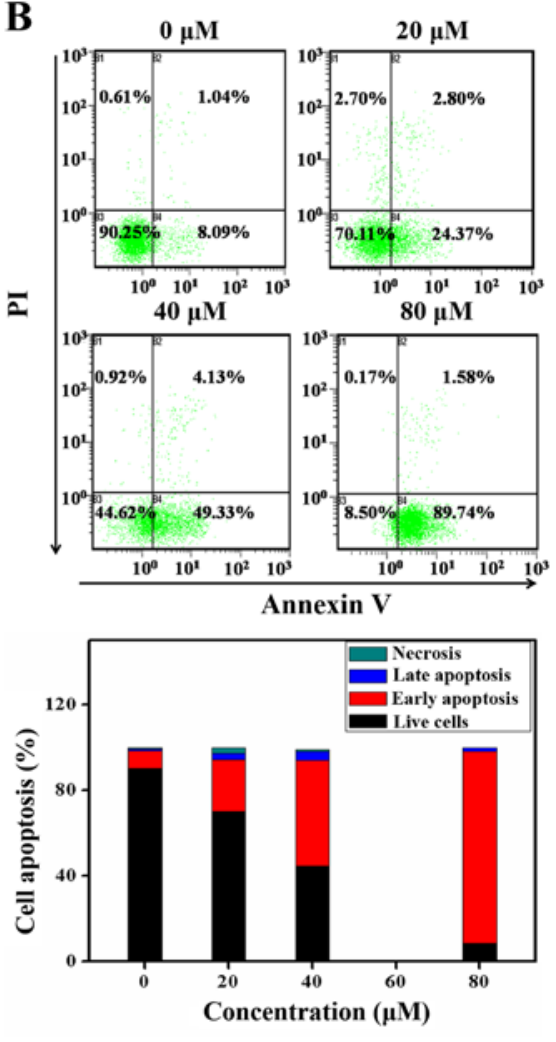

Figure 2. Mechanism of induction of cordycepin on SGC-7901. (A) Cells were treated with cordycepin, then stained by DAPI. Cordycepin ruptured the cell membranes resulting in inducing the nuclear condensation and DNA fragmentation. (B) SGC-7901 cells were treated with cordycepin then stained with Annexin V/PI. The lower right quadrant shows $\mathrm{V}^{+} / \mathrm{PI}^{-}$Annexin, the upper right quadrant shows $\mathrm{V}^{+} / \mathrm{PI}^{+} \mathrm{Annexin}$ cells. Each bar represented the percentage (mean $\pm \mathrm{SD}$ of triplicate determinations) of both Annexin $\mathrm{V}^{+} / \mathrm{PI}^{-}$and $\mathrm{V}^{+} / \mathrm{PI}^{+}$Annexin cells. "Values significantly different from control ( $\mathrm{n}=3$; $\mathrm{P}<0.05$ ). (C) Cytoplasmic proteins were fractionated by 8-12\% SDS-PAGE, transferred onto nitrocellulose membranes, and subjected to ECL detection analysis. The equivalent loading of proteins in each well was confirmed by $\beta$-actin and Ponceau staining. 


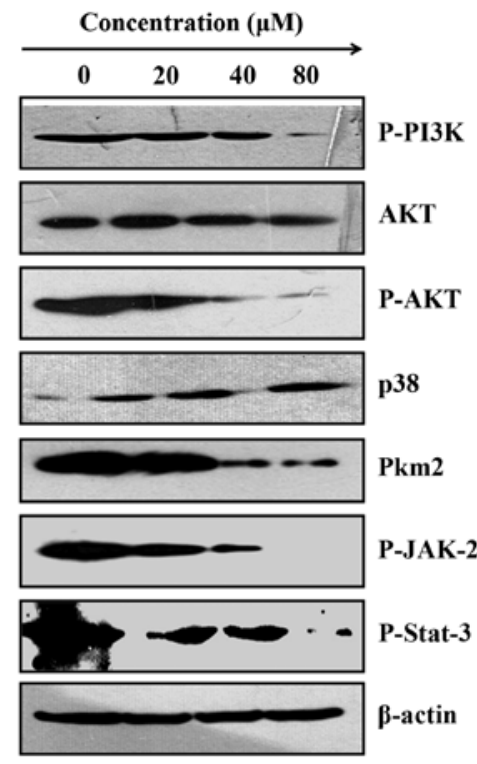

Figure 3. Cordycepin mediates SGC-7901 cell death by phosphorylated PI3K/ AKT, JAK2/STAT-3 and activated P38 proteins. Cytoplasmic proteins were fractionated by $10 \%$ SDS-PAGE, transferred onto nitrocellulose membranes, and subjected to the ECL detection analysis. out and results showed that cordycepin inhibited drastically the protein expression of $\mathrm{A}_{3} \mathrm{AR}$ expression (Fig. 2C). Based on this we suggest that inhibition of $A_{3} A R$ is likely to play a role in the induction of apoptosis mediated by cordycepin on SGC-7901 cells.

Cordycepin exerts anti-inflammatory activity in SGC-7910 cells by phosphorylated STAT-3/JAK2. The Jak-Stat cascade proteins are essential for inflammatory as well as immune responses of anticancer agents (33). The impacts of cordycepin on the Jak-Stat protein expression was probed with the help of western blot analysis. The findings brought to light that cordycepin improved the phosphorylation of Jak2 and Stat3 proteins in SGC-7901 cells (Fig. 3). This is clearly due to the potential of cordycepin to translocate Stat 3 and Jak2 from the cytoplasm into the nucleus leading to the initiation of the gene expression of pro-inflammatory response.

Cordycepin induces apoptosis in SGC-7901 cells by collapse of mitochondrial membrane potential and generation of reactive oxygen species. To validate whether mitochondrial events were involved in induction of apoptosis, flow cytometry of
A

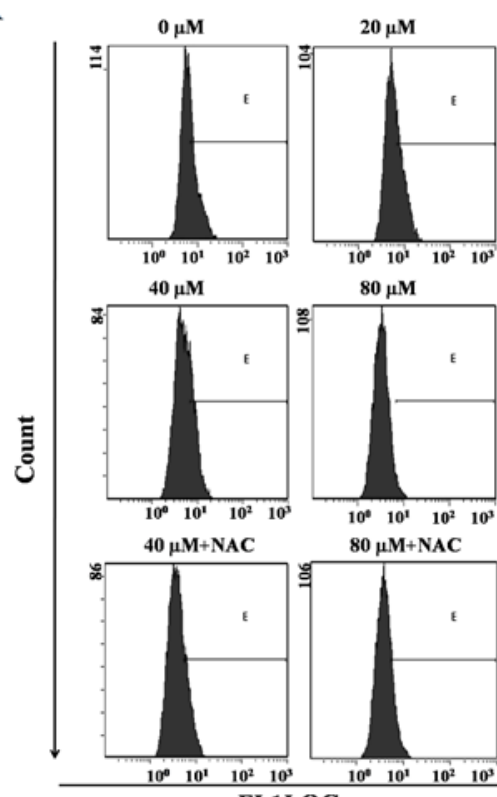

FL1LOG

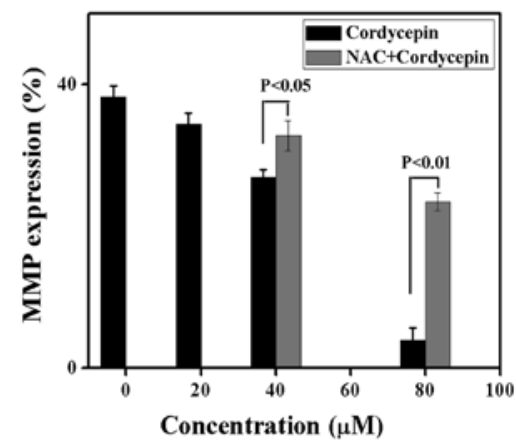

B

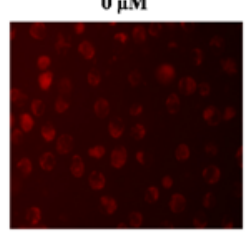

$40 \mu \mathrm{M}$
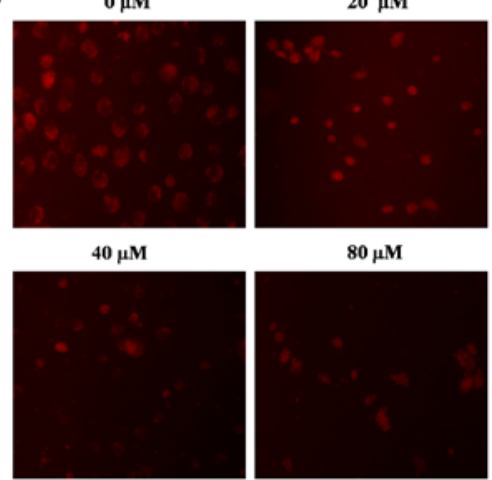

$80 \mu \mathrm{M}$

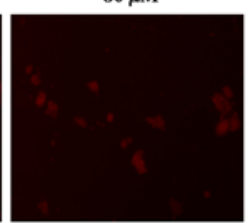

$\mathrm{C}$

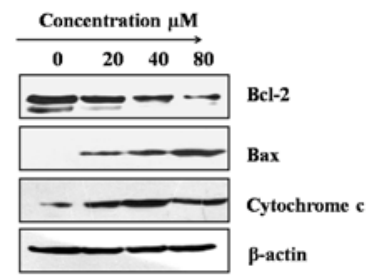

Figure 4. Cordycepin represses mitochondrial membrane potential in SGC-7901 cells. (A) SGC-7901 cells were treated with or without cordycepin in absence or presence of NAC $3 \mathrm{mM}$. The data shown are representative of three independent experiments with similar results. $\mathrm{P}<0.05$; and $\mathrm{P}<0.01$ comparing cordycepin-treated cells with the cordycepin and NAC (inhibitor)-treated cells. (B) SGC-7901 cells were observed under fluorescence microscope: treatment with cordycepin induces SGC-7901 cell membrane changes and change induces lost of metabolites from MM leading to their collapse. (C) Relative protein expression regulation of mitochondrial membrane potential. Cytochrome $c$, Bax and Bcl-2 protein were examined to confirm the MM collapse by cordycepin on SGC-7901 cells. 


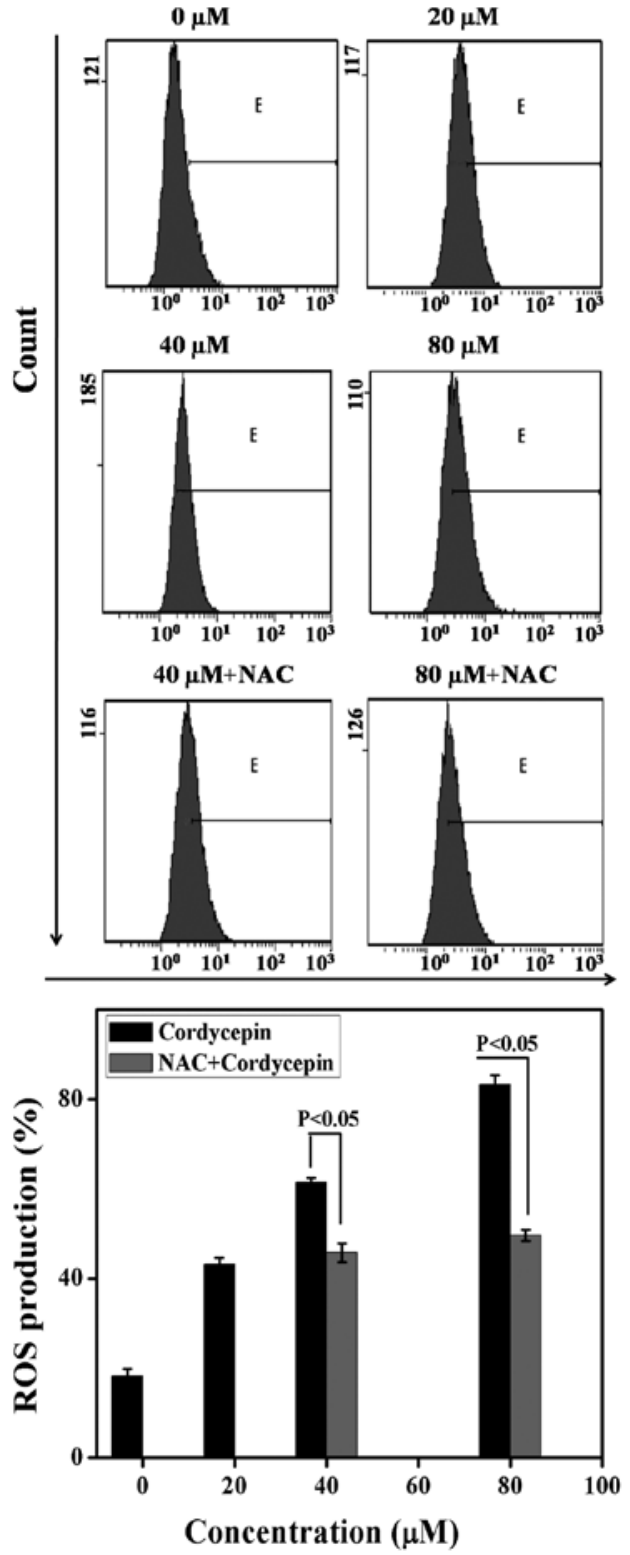

Figure 5. Cordycepin induces reactive oxygen production in SGC-7901 cells. SGC-7901 cells were treated with or without cordycepin in the absence or presence of NAC $3 \mathrm{mM}$. The data shown are representative of three independent experiments with similar results. $\mathrm{P}<0.05$ comparing cordycepin-treated cells with the cordycepin and NAC (inhibitor)-treated cells.

rhodamine 123 staining and western blot analysis was carried out. As depicted in Fig. 4A, during the unavailability of NAC, the mitochondrial membrane potential expression is minimized to a significant extent. On the other hand, the mitochondrial membrane potential expression was restored in the availability of NAC upon utilization of cordycepin $(0,20,40$ and $80 \mu \mathrm{M})$ for $24 \mathrm{~h}$, recommending that mitochondrial were taking part in cordycepin induced SGC-7901 cell apoptosis. To confirm our findings immunoflorescence of Rhodamine were further processed. As seen in Fig. 4B, cordycepin induce SC-7901 cells morphology changes due to the lost of mitochondrial membrane metabolite resulting in their collapse.

Moreover, to develop more understanding of the process by which cordycepin decreased mitochondrial membrane potential, western blot analysis is carried out for the purpose of validating the level of cytochrome $c$, Bax, and Bcl-2 to obtain more insight into cell apoptosis. The findings suggest that cordycepin resulted in release of cytochrome $c$ from the mitochondrial membrane which is the major factor in the development of apoptosomes, which trigger the activation of Bax and deactivates Bcl-2 (Fig. 4C).

Previous study on cordycepin shows that ROS is linked to a collapse of mitochondrial membrane potential (34). We carried out an analysis of the production of intracellular ROS level with the help of flow cytometry to evaluate whether apoptosis was caused by cordycepin. Specifically, the cells treated with cordycepin were loaded with the fluorescent probes DCF-DA to gauge the $\mathrm{H}_{2} \mathrm{O}_{2}$ in the availability as well as unavailability of NAC and incubated for $24 \mathrm{~h}$. The findings show that, in the absence of NAC, the ROS values are $18.22 \pm 1.52,43.14 \pm 1.52,61.39 \pm 1.73$ and $83.23 \pm 2.075$ upon 0 , 20, 40 and $80 \mu \mathrm{M}$ of cordycepin, correspondingly. On the other hand, in the presence of NAC, the generation of ROS expression are $45.72 \pm 2.08$ and $49.57 \pm 1.25$ via utilization of 40 , and $80 \mu \mathrm{M}$ cordycepin correspondingly denoting the role of NAC in minimizing $\mathrm{H}_{2} \mathrm{O}_{2}$ (Fig. 5). These results validate the role of cordycepin in the generation of oxidative stress in SGC-7901 cells.

Cordycepin induces cell cycle arrest at $S$ phase. To validate whether the inhibition of Akt can influence SGC-7901 cell cycle arrest, flow cytometry was carried out. In the four cell cycle phases, the $\mathrm{S}$ phase, $\mathrm{M}$ phase, G1, and G2 phase, the treatment of SGC-7901 cell line with $0,20,40$, and $80 \mu \mathrm{M}$ for $24 \mathrm{~h}$ induce an increase in the $\mathrm{S}$ phase percentages to $45.29 \pm 2.13,53.315 \pm 2.54,71.092 \pm 2.24$ and 71.203 \pm 3.1 . These concentrations also resulted in the decrease of the G0/G1 and $\mathrm{G} 2 / \mathrm{M}$ phase percentages. Based on the above evidence, we confirmed that cordycepin induces SGC-7901 cell cycle arrest at the $\mathrm{S}$ phase (Fig. 6A). To further delineate and validate the process of the SGC-7901 cell cycle arrest by cordycepin, the protein expression of cyclin E, CDK1 and CDK2, cyclin D1, p53, p21 MDM2, and pRb proteins were explored by western blot analysis. Fig. 6B suggests that cordycepin suppressed significantly the expression of cyclin E, CDK1, CDK2, MDM2, cyclin D1, as well as phosphorylated pRb. At the same time, cordycepin augmented the p53 and p21 expression. These findings indicated that cordycepin induces SGC-7901 cell progression at $\mathrm{S}$ phase (Fig. 6A).

\section{Discussion}

It has been reported that there are two types of gastric carcinoma cells EBV-positive and EBV-negative. Recently, it has been suggested that EBV-positive gastric carcinomas have distinct molecular characteristics in comparison with EBV-negative gastric carcinomas (35). Regarding this we evaluated the cytotoxicity of cordycepin against SGC-7901 (EBV-negative) to compare with a previous study (18).

To shed light on the cytotoxicity mechanism trigged by cordycepin, we attempted to identify the molecular mechanisms involved in cordycepin apoptosis and cell cycle in SGC-7901. To our knowledge, this is the first report showing the mechanism behind with cordycepin induced gastric cancer cell cytotoxicity. Cordycepin not only induced SGC-7901 cells 
A
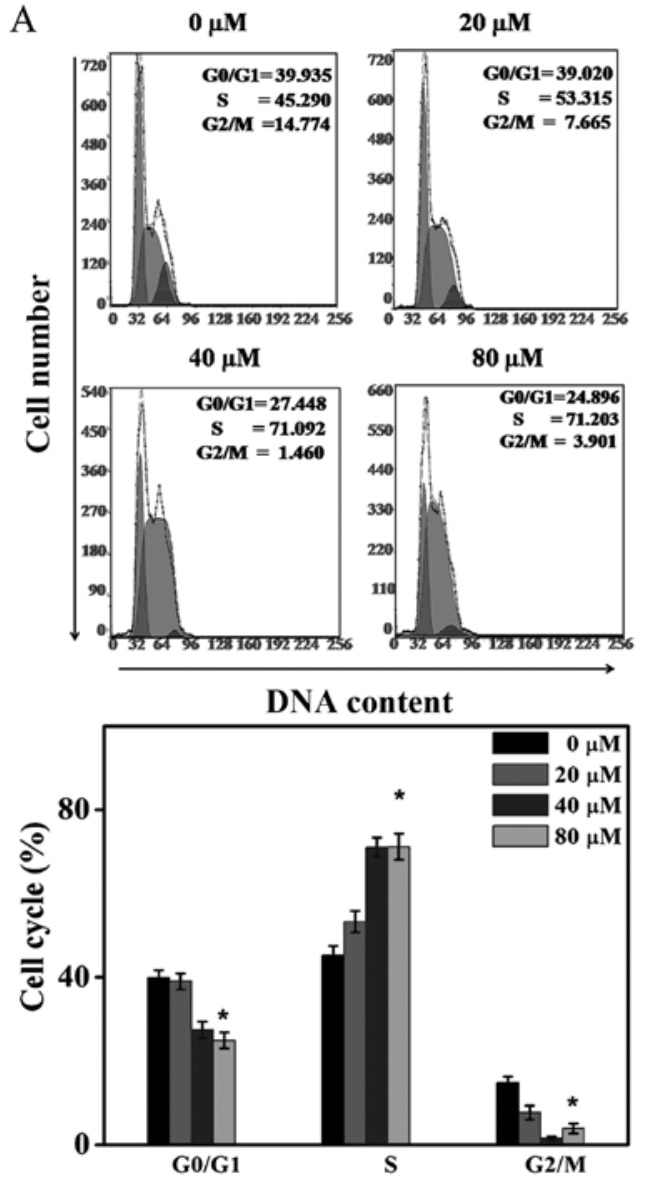

B

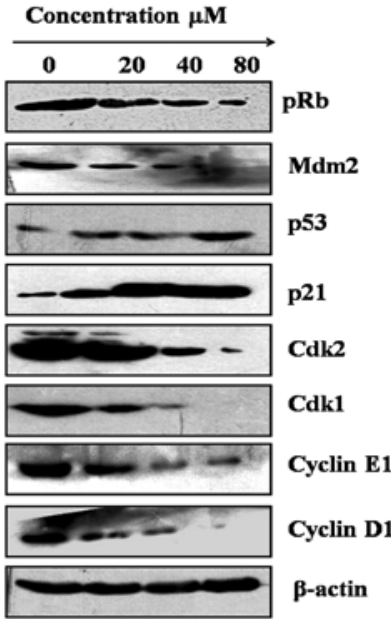

Figure 6. Effect of cordycepin on SGC-7901 cell cycle distribution. (A) SGC-7901 cells were treated with 0, 20,40 and $80 \mu \mathrm{M}$ of cordycepin for $24 \mathrm{~h}$ and then they were stained with PI for flow cytometric analysis. Histograms show number of cells/channel (y-axis) vs. DNA content (x-axis). The values indicate the percentage of cells in the indicated phases of the cell cycle. The data shown are representative of three independent experiments with similar results. "P<0.05; compared with the control. (B) Cytoplasmic proteins were fractionated by $8-12 \%$ SDS-PAGE, transferred onto nitrocellulose membranes, and subjected to the ECL detection analysis. The equivalent loading of proteins in each well was confirmed by $\beta$-actin and Ponceau staining.

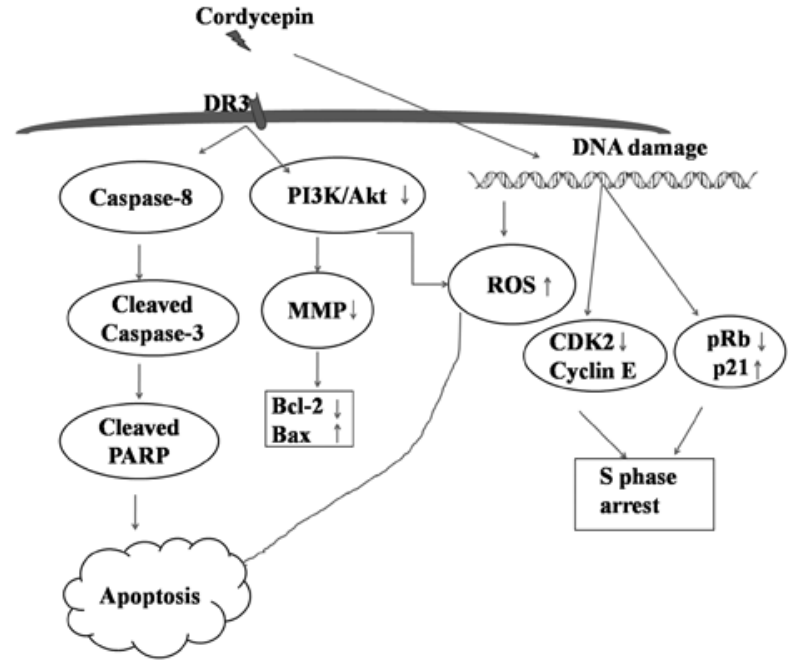

Figure 7. Hypothetical mechanism of action of cordycepin on SGC-7901 cells. Cordycepin induces activation of DR3, which activated caspase-8/3 and cleaved PRAP consequently induce SGC-7901 cell death by apoptosis. Simultaneous activation of DR3 by cordycepin drives the phosphorylation of PI3K/AKT signaling resulting in the collapse to MMP that drives inhibition of the complex Bcl-2/Bax. Cordycepin disrupts SGC-7901 cell DNA causing oxidative stress in SGC-7901 cells therefore generating hyper production of ROS, thus the DNA damage induced inhibition of the complex cyclin E/ CDK2 and increased the expression of p21 consequently inducing SGC-7901 cell cycle arrest at $\mathrm{S}$ phase. growth changes but also triggers their morphology changes in line with initial reports $(36,37)$. This is due to the unique resemblance of cordycepin to disturb the cell membranes leading to a DNA damage influencing cell death by apoptosis. This is additionally validated through the evaluation of the expression of pro- and anti-apoptotic Bcl-2 proteins during SGC-7901 cell apoptosis (38). The imbalance between these proteins resulted in the loss in the mitochondrial trans-membrane potential $(\Delta \psi \mathrm{m})$ leading to cell death by apoptosis (38). Apoptosis can take place due to death receptors such as DR3. When activated by external stimuli DR3 can eliminate the activation of caspase- 8 resulting in activating the downstream caspase9/3. Cordycepin activates the DR3 and eliminates SGC-7901 celldeath by apoptosis. In the same manner, cordycepin releases cytochrome $c$ a major factor in the development of apoptosomes that activates caspase- 3 , which drives cleavage of the PARP and that is why it induces cell death by apoptosis. These findings are similar to previous reports (14). Moreover, it has been reported that cordycepin induces inhibition of thyroid carcinoma cells through suppressing the expression of $\mathrm{A}_{3} \mathrm{AR}$ (39). Herein, treatment of SGC-7901 cell line induced inhibition of $\mathrm{A}_{3} \mathrm{AR}$ protein expression thus we suggest that cordycepin induces SGC-7901 cell apoptosis mainly by suppression of $\mathrm{A}_{3} \mathrm{AR}$. 
Activation of Akt affects cell growth and progression. Earlier, cordycepin was observed to assert anticancer impact by minimizing PI3K/Akt pathway (21). Herein our findings brought to light that cordycepin downregulated PI3K/Akt that was linked to SGC-7901 cell apoptosis. Furthermore, our current study indicates that the anti-apoptotic impacts of Akt on gastric cancer cell death is linked to the generation of ROS. These findings are similar to a previous study and confirmed the elimination of PI3K/Akt signaling by cordycepin (20). Moreover, pyruvate protein kinase isoform M2 (PKM2) imparts a leading role in the nucleus phosphorylation of Akt and PI3K (31). In this way, inhibition of PKM2 expression by cordycepin triggers the phosphorylation of PI3K/Akt that is ascribed to the efficacy of cordycepin to downregulate PI3K/ Akt which is presented to be involved in the secretion of PKM2 in SGC-7910 cells. Furthermore, cordycepin phosphorylated STAT-3 and JAK-2 protein result from their translocation from the cytoplasm to the nucleus which assists in expression of pro-inflammatory genes (40).

The molecular mechanism of cordycepin is further validated by the benchmarking the SGC-7901 cell cycle arrest. The molecular process of the cancer cell cycle regulation is interfered by the modifications in the major checkpoint of genes (41). Cordycepin drive SGC-7901 cell cycle arrested at the $\mathrm{S}$ phase. This is due to the potential of cordycepin to downregulate the protein expression of the complex CDK2/ cyclin $\mathrm{E}$ in addition to upregulation of the p21 and phosphorylated $\mathrm{pRb}$ proteins that are playing a crucial role in promoting the cell progression with the help of $\mathrm{S}$ phase (42). Furthermore, the DNA damage in gastric cancer results in the inhibition of MDM2 and improvement of p53 (43). This is where we can presume that the inhibition of MDM2 protein expression and increase of p53 might play their parts in the cell cycle arrest induced by cordycepin in SGC-7901 cells.

This study, revealed the process by which cordycepin functions as an effectual anti-proliferating agent to deal with SGC-7901 gastric cancer cell line. Apoptosis induced by cordycepin was linked to the mitochondrial extrinsic pathways mainly by activation of DR3, inhibition of $\mathrm{A}_{3} \mathrm{AR}$ and the collapse of mitochondrial membrane potential. In addition, cordycepin promotes phosphorylation of PI3K/Akt that results into the generation of ROS in addition to cell cycle arrest at $\mathrm{S}$ phase (Fig. 7). Considered collectively, these findings suggest cordycepin as an efficient drug against gastric cancer.

However, cordycepin being an adenosine analogue must affect the cells with adenosine receptors, more studies are required to investigate the effect and its molecular mechanism on cordycepin in other cell lines.

\section{Acknowledgements}

This study was supported by Ministry of Science and Technology (no. 2016YFE0128500), Jilin Provincial Science and Technology Department (20130521010JH, 20150101187JC, and 20150414007GH), Jilin Province Education Department (2015-526, 2015-551); the Fundamental Research Funds for the Central Universities (2412015ZH005, 2412016KJ037, 130017507, 130028633). University S\&T Innovation Platform of Jilin Province for Economic Fungi (no. 2014B-1). National Natural Science Foundation of China (nos. 30871301 and
30700827), and the Program for Introducing Talents to Universities (no. B07017).

\section{References}

1. Zhao EH, Ling TL and $\mathrm{Cao} \mathrm{H}$ : Current status of surgical treatment of gastric cancer in the era of minimally invasive surgery in China: Opportunity and challenge. Int J Surg 28: 45-50, 2016.

2. Oba K, Paoletti X, Bang YJ, Bleiberg H, Burzykowski T, Fuse N, Michiels S, Morita S, Ohashi Y, Pignon JP, et al; GASTRIC (Global Advanced/Adjuvant Stomach Tumor Research International Collaboration) Group: Role of chemotherapy for advanced/recurrent gastric cancer: An individual-patient-data meta-analysis. Eur J Cancer 49: 1565-1577, 2013.

3. Kim HS, Kim JH, Kim JW and Kim BC: Chemotherapy in elderly patients with gastric cancer. J Cancer 7: 88-94, 2016.

4. Moehler M, Delic M, Goepfert K, Aust D, Grabsch HI, Halama N, Heinrich B, Julie C, Lordick F, Lutz MP, et al: Immunotherapy in gastrointestinal cancer: Recent results, current studies and future perspectives. Eur J Cancer 59: 160-170, 2016.

5. Chen W, Zhao Z, Li L, Wu B, Chen SF, Zhou H, Wang Y and Li YQ: Hispolon induces apoptosis in human gastric cancer cells through a ROS-mediated mitochondrial pathway. Free Radic Biol Med 45: 60-72, 2008.

6. Nishiyama M, Yoshino S, Matsui H, Sakamoto K, Suzuki N, Tamesa T, Takeda S, Ueno T, Hazama S and Oka M: A case of metachronous liver metastasis from gastric cancer successfully treated with hepatectomy. Gan To Kagaku Ryoho 41: 2352-2354, 2014 (In Japanese).

7. Cui F, Zan X, Li Y, Sun W, Yang Y and Ping L: Grifola frondosa glycoprotein GFG-3a arrests S phase, alters proteome, and induces apoptosis in human gastric cancer cells. Nutr Cancer 68: 267-279, 2016.

8. Wang F, Yin P, Lu Y, Zhou Z, Jiang C, Liu Y and Yu X: Cordycepin prevents oxidative stress-induced inhibition of osteogenesis. Oncotarget 6: 35496-35508, 2015.

9. Tianzhu Z, Shihai Y and Juan D: The effects of cordycepin on ovalbumin-induced allergic inflammation by strengthening Treg response and suppressing Th17 responses in ovalbuminsensitized mice. Inflammation 38: 1036-1043, 2015.

10. Hwang JH, Joo JC, Kim DJ, Jo E, Yoo HS, Lee KB, Park SJ and Jang IS: Cordycepin promotes apoptosis by modulating the ERK-JNK signaling pathway via DUSP5 in renal cancer cells. Am J Cancer Res 6: 1758-1771, 2016.

11. Shao LW, Huang LH, Yan S, Jin JD and Ren SY: Cordycepin induces apoptosis in human liver cancer HepG2 cells through extrinsic and intrinsic signaling pathways. Oncol Lett 12: 995-1000, 2016.

12. Baig S, Seevasant I, Mohamad J, Mukheem A, Huri HZ and Kamarul T: Potential of apoptotic pathway-targeted cancer therapeutic research: Where do we stand? Cell Death Dis 7: e2058, 2016.

13. Tian X, Li Y, Shen Y, Li Q, Wang Q and Feng L: Apoptosis and inhibition of proliferation of cancer cells induced by cordycepin. Oncol Lett 10: 595-599, 2015.

14. Lee SY, Debnath T, Kim SK and Lim BO: Anti-cancer effect and apoptosis induction of cordycepin through DR3 pathway in the human colonic cancer cell HT-29. Food Chemical Toxicol 60: 439-447, 2013.

15. Ashkenazi A and Dixit VM: Death receptors: Signaling and modulation. Science 281: 1305-1308, 1998 .

16. Williams JO, Wang EC, Lang D and Williams AS: Characterization of death receptor 3-dependent aortic changes during inflammatory arthritis. Pharmacol Res Perspect 4: e00240, 2016.

17. Calder CJ and Wang EC: An essential role for death receptor 3 in experimental autoimmune uveoretinitis. Ocul Immunol Inflamm 20: 212-214, 2012.

18. Ryu E, Son M, Lee M, Lee K, Cho JY, Cho S, Lee SK, Lee YM, Cho $\mathrm{H}$, Sung GH, et al: Cordycepin is a novel chemical suppressor of Epstein-Barr virus replication. Oncoscience 1: 866-881, 2014.

19. Du Y, Yu J, Du L, Tang J and Feng WH: Cordycepin enhances Epstein-Barr virus lytic infection and Epstein-Barr virus-positive tumor treatment efficacy by doxorubicin. Cancer Lett 376: 240-248, 2016.

20. Pan BS, Wang YK, Lai MS, Mu YF and Huang BM: Cordycepin induced MA-10 mouse Leydig tumor cell apoptosis by regulating p38 MAPKs and PI3K/AKT signaling pathways. Sci Rep 5: 13372, 2015. 
21. Jeong JW, Jin CY, Park C, Han MH, Kim GY, Moon SK, Kim CG Jeong YK, Kim WJ, Lee JD, et al: Inhibition of migration and invasion of LNCaP human prostate carcinoma cells by cordycepin through inactivation of Akt. Int J Oncol 40: 1697-1704, 2012.

22. Ye Y, Ge YM, Xiao MM, Guo LM, Li Q, Hao JQ, Da J, Hu WL, Zhang XD, Xu J, et al: Suppression of SHIP2 contributes to tumorigenesis and proliferation of gastric cancer cells via activation of Akt. J Gastroenterol 51: 230-240, 2016.

23. Zheng T, Meng X, Wang J, Chen X, Yin D, Liang Y, Song X, Pan S, Jiang $\mathrm{H}$ and Liu L: PTEN- and p53-mediated apoptosis and cell cycle arrest by FTY720 in gastric cancer cells and nude mice. J Cell Biochem 111: 218-228, 2010.

24. Martin DA and Elkon KB: Mechanisms of apoptosis. Rheum Dis Clin North Am 30: vii 441-vii454, 2004.

25. Zhao Z, Han F, Yang S, Wu J and Zhan W: Oxamate-mediated inhibition of lactate dehydrogenase induces protective autophagy in gastric cancer cells: Involvement of the Akt-mTOR signaling pathway. Cancer Lett 358: 17-26, 2015.

26. Panieri E and Santoro MM: ROS homeostasis and metabolism: A dangerous liason in cancer cells. Cell Death Dis 7: e2253, 2016.

27. Chen Y, Zhang H, Zhou HJ, Ji W and Min W: Mitochondrial redox signaling and tumor progression. Cancers (Basel) 8: 8, 2016.

28. Khan M, Rasul A, Yi F, Zhong L and Ma T: Jaceosidin induces p53-dependent G2/M phase arrest in U87 glioblastoma cells. Asian Pac J Cancer Prev 12: 3235-3238, 2011.

29. Singh SS, Yap WN, Arfuso F, Kar S, Wang C, Cai W, Dharmarajan AM, Sethi G and Kumar AP: Targeting the PI3K/ Akt signaling pathway in gastric carcinoma: A reality for personalized medicine? World J Gastroenterol 21: 12261-12273, 2015.

30. Porquet N, Poirier A, Houle F, Pin AL, Gout S, Tremblay PL, Paquet ER, Klinck R, Auger FA and Huot J: Survival advantages conferred to colon cancer cells by E-selectin-induced activation of the PI3K-NFxB survival axis downstream of death receptor-3. BMC Cancer 11: 285, 2011.

31. Chen G, Feng W, Zhang S, Bian K, Yang Y, Fang C, Chen M, Yang $\mathrm{J}$ and Zou X: Metformin inhibits gastric cancer via the inhibition of HIF1 $\alpha /$ PKM2 signaling. Am J Cancer Res 5 : $1423-1434,2015$

32. Fishman P, Cohen $S$ and Bar-Yehuda S: Targeting the A3 adenosine receptor for glaucoma treatment (Review). Mol Med Rep 7: 1723-1725, 2013.
33. Khanna P, Chua PJ, Bay BH and Baeg GH: The JAK/STAT signaling cascade in gastric carcinoma (Review). Int J Oncol 47: 1617-1626, 2015

34. Lee HH, Park C, Jeong JW, Kim MJ, Seo MJ, Kang BW, Park JU, Kim GY, Choi BT, Choi YH, et al: Apoptosis induction of human prostate carcinoma cells by cordycepin through reactive oxygen species-mediated mitochondrial death pathway. Int J Oncol 42: 1036-1044, 2013

35. He D, Zhang YW, Zhang NN, Zhou L, Chen JN, Jiang Y and Shao CK: Aberrant gene promoter methylation of p16, FHIT, CRBP1, WWOX, and DLC-1 in Epstein-Barr virus-associated gastric carcinomas. Med Oncol 32: 92, 2015.

36. Tao X, Ning Y, Zhao X and Pan T: The effects of cordycepin on the cell proliferation, migration and apoptosis in human lung cancer cell lines A549 and NCI-H460. J Pharm Pharmacol 68: 901-911, 2016.

37. Li Y, Li R, Zhu S, Zhou R, Wang L, Du J, Wang Y, Zhou B and Mai L: Cordycepin induces apoptosis and autophagy in human neuroblastoma SK-N-SH and BE (2) -M17 cells. Oncol Lett 9: 2541-2547, 2015.

38. Qiao L and Wong BC: Targeting apoptosis as an approach for gastrointestinal cancer therapy. Drug Resist Updat 12: 55-64, 2009.

39. Chen Y, Chen YC, Lin YT, Huang SH and Wang SM: Cordycepin induces apoptosis of CGTH W-2 thyroid carcinoma cells through the calcium-calpain-caspase 7-PARP pathway. J Agric Food Chem 58: 11645-11652, 2010.

40. Zearfoss NR, Alarcon JM, Trifilieff P, Kandel E and Richter JD: A molecular circuit composed of CPEB-1 and c-Jun controls growth hormone-mediated synaptic plasticity in the mouse hippocampus. J Neurosci 28: 8502-8509, 2008.

41. Viallard JF, Lacombe F, Belloc F, Pellegrin JL and Reiffers J: Molecular mechanisms controlling the cell cycle: Fundamental aspects and implications for oncology. Cancer Radiother 5: 109-129, 2001 (In French).

42. Liao Y, Ling J, Zhang G, Liu F, Tao S, Han Z, Chen S, Chen Z and Le H: Cordycepin induces cell cycle arrest and apoptosis by inducing DNA damage and up-regulation of p53 in leukemia cells. Cell Cycle 14: 761-771, 2015.

43. Choi HS, Seo HS, Kim JH, Um JY, Shin YC and Ko SG: Ethanol extract of paeonia suffruticosa Andrews (PSE) induced AGS human gastric cancer cell apoptosis via fas-dependent apoptosis and MDM2-p53 pathways. J Biomed Sci19: 82, 2012. 